\title{
Duality of capacities and Sobolev extendability in the plane
}

\author{
Yi Ru-Ya Zhang'
}

Received: 30 January 2020 / Accepted: 20 January 2021 / Published online: 22 February 2021

(c) The Author(s) 2021

\begin{abstract}
We reveal relations between the duality of capacities and the duality between Sobolev extendability of Jordan domains in the plane, and explain how to read the curve conditions involved in the Sobolev extendability of Jordan domains via the duality of capacities. Finally as an application, we give an alternative proof of the necessary condition for a Jordan planar domain to be $W^{1, q}$-extension domain when $2<q<\infty$.
\end{abstract}

Keywords Capacity $\cdot$ Sobolev extension domain

Mathematics Subject Classification $30 \mathrm{C} 85 \cdot 46 \mathrm{E} 35$

\section{Introduction}

Let $\Omega$ be a Jordan domain in the plane, that is, $\Omega$ is the bounded connected component of $\mathbb{R}^{2} \backslash \gamma_{0}$ for a Jordan curve

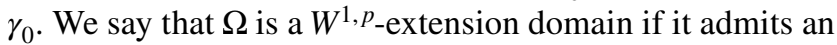
extension operator $E: W^{1, p}(\Omega) \rightarrow W^{1, p}\left(\mathbb{R}^{2}\right)$ such that, there exists a constant $C \geq 1$ so that for every $u \in W^{1, p}(\Omega)$ we have

$\|E u\|_{W^{1, p}\left(\mathbb{R}^{2}\right)} \leq C\|u\|_{W^{1, p}(\Omega)}$

and $\left.E u\right|_{\Omega}=u$. Here we define the Sobolev space $W^{1, p}(\Omega)$, $1 \leq p \leq \infty$, as

$W^{1, p}(\Omega)=\left\{u \in L_{\text {loc }}^{1}(\Omega): \nabla u \in L^{p}\left(\Omega, \mathbb{R}^{2}\right)\right\}$,

where $\nabla u$ denotes the distributional gradient of $u$. The (semi)-norm in $W^{1, p}(\Omega)$ that we consider here is

$\|u\|_{W^{1, p}(\Omega)}=\|\nabla u\|_{L^{p}(\Omega)}$.

The following result was proven in [15].

Dedicated to the 60th birthday of Pekka Koskela.

Yi Ru-Ya Zhang

yizhang3@ethz.ch

1 Department of Mathematics, ETH Zürich, Rämistrasse 101, 8092 Zurich, Switzerland
Theorem 1.1 Let $\Omega \subset \mathbb{C}$ be a Jordan domain. Then it is a $W^{1, p}$-extension domain if and only if $\tilde{\Omega}:=\mathbb{C} \backslash \bar{\Omega}$ is a $W^{1, q}$ -extension domain for $1<p<\infty$ and $q=\frac{p}{p-1}$.

This duality of extendability is indeed hinted by the following duality of capacities in the plane, which originally comes from [23] and can be applied to show, for instance, Uniformization Theorem [18]. Recall that for a given pair of continua $E, F \subset \bar{\Omega} \subset \mathbb{R}^{2}$ and $1<p<\infty$, one defines the $p$-capacity between $E$ and $F$ in $\Omega$ as

$\operatorname{Cap}_{p}(E, F ; \Omega)=\inf \left\{\|\nabla u\|_{L^{p}(\Omega)}^{p}: u \in \Delta(E, F ; \Omega)\right\}$,

where $\Delta(E, F ; \Omega)$ denotes the class of all $u \in W^{1, p}(\Omega)$ that are continuous in $\Omega \cup E \cup F$ and satisfy $u=1$ on $E$, and $u=0$ on $F$. Observe that by definition the $p$-capacity is increasing with respect to $E, F, \Omega$.

Theorem 1.2 ([1, 19]) Let $\Omega \subset \hat{\mathbb{C}}$ be a Jordan domain enclosed by four arcs $\gamma_{1}, \gamma_{2}, \gamma_{3}$ and $\gamma_{4}$ counterclockwise. Then we have

$\left(\operatorname{Cap}_{p}\left(\gamma_{1}, \gamma_{3} ; \Omega\right)\right)^{\frac{1}{p}}\left(\operatorname{Cap}_{q}\left(\gamma_{2}, \gamma_{4} ; \Omega\right)\right)^{\frac{1}{q}}=1$

for $1<p<\infty$ and $q=\frac{p}{p-1}$.

To see how the duality in Theorem 1.1 comes from (1.1), suppose $\Omega$ is a $W^{1, q}$-extension Jordan domain with $2<q<\infty$ and write 
$\partial \Omega=\bigcup_{i=1}^{4} \gamma_{i}$

as in Theorem 1.2. By denoting $\tilde{\Omega}$ the complementary domain of $\Omega$, since $\Omega$ is a $W^{1, q}$-extension Jordan domain, there exists a constant $C_{0} \geq 1$ so that

$\operatorname{Cap}_{q}\left(\gamma_{2}, \gamma_{4} ; \tilde{\Omega}\right) \leq C_{0} \operatorname{Cap}_{q}\left(\gamma_{2}, \gamma_{4} ; \Omega\right)$

Then by applying Theorem 1.2 to both $\Omega$ and $\tilde{\Omega}$ (together with a technical lemma [15, Lemma 2.1] saying that we can always swap an unbounded domain with compact boundary to a bounded domain (and vice versa) with the same extendability), we conclude that

$\operatorname{Cap}_{p}\left(\gamma_{1}, \gamma_{3} ; \Omega\right) \leq C\left(p, C_{0}\right) \operatorname{Cap}_{p}\left(\gamma_{1}, \gamma_{3} ; \tilde{\Omega}\right)$.

By the arbitrariness of $\gamma_{1}, \gamma_{3} \subset \partial \Omega$, we conclude the extendability of $p$-capacity functions in $\tilde{\Omega}$, i.e. those functions in $W^{1, p}(\tilde{\Omega})$ take value 0 and 1 on two distinct subarcs of $\partial \Omega$, respectively. Then by the fact the exdentability of $p$-capacity functions in a planar Jordan domain implies the extendability of $W^{1, p}$-functions in that domain, we conclude the desired duality of Sobolev extendability. This fact was proven in [15] via an indirect method, and also see the recent paper [13] for further information.

To be more specific, for $1<p<2$ in [15] we first show that, if every $p$-capacity function in a Jordan domain $\Omega$ is extendable, then its complementary domain $\tilde{\Omega}$ is $(2-p)$ -subhyperbolic, i.e. for $1<p<2$ and every $z_{1}, z_{2} \in \tilde{\Omega}$, there exist a constant $C_{1}>0$ and a curve $\gamma \subset \tilde{\Omega}$ joining $z_{1}$ and $z_{2}$ such that

$\int_{\gamma} \operatorname{dist}(z, \partial \Omega)^{1-p} \mathrm{~d} s(z) \leq C_{1}\left|z_{1}-z_{2}\right|^{2-p}$.

Then via this curve condition, we constructed an extension operator for all functions in $W^{1, p}(\Omega)$.

The curve condition (1.2) has been studied for a long time, up to notational change on the exponent. For example it can be used to characterize Lip $_{\alpha}$-extension domain; see e.g. [5, 16]. Also it appears in the characterization of $W^{1, q}$-extension domains with $q>2$; see for instance [3, $12,20]$.

In this paper, we show the relation between (1.2) and the duality of capacities (1.1). Towards this, let us introduce some terminology. Recall that the left-hand side of (1.2) is called the $p$-subhyperbolic length of $\gamma$ for $1<p<2$, and accordingly we define the $p$-subhyperbolic distance between $z_{1}, z_{2}$, denoted by $d_{p}\left(z_{1}, z_{2}\right)$, via taking infimum of the $p$-subhyperbolic length among all the curves joining $z_{1}, z_{2}$ in $\Omega$. For more properties of this metric we refer to [20].

For $1<p \leq 2$ and $z_{1}, z_{2} \in \Omega$, the $p$-capacity between $z_{1}$ and $z_{2}$ in $\Omega$ is defined as
$d_{\mathrm{Cap}_{p}}\left(z_{1}, z_{2} ; \Omega\right)=\inf _{\gamma} \operatorname{Cap}_{p}(\gamma, \partial \Omega ; \Omega)$,

where the infimum is again taken over all the curves connecting $z_{1}$ and $z_{2}$ inside $\Omega$. We remark that the triangle inequality for this metric follows naturally from the subadditivity of capacity; see e.g. [4, Theorem 2 (vii), Chapter 4.7].

The theorems below indicate the relation between the $p$-capacity metric and the $(2-p)$-subhyperbolic metric for $1<p<2$ in the plane.

Theorem 1.3 Let $1<p<2$ and $\Omega \subset \mathbb{C}$ be a Jordan domain and $p \in(1,2)$, and $z_{1}, z_{2} \in \Omega$. Then for the hyperbolic geodesic $\gamma$ joining $z_{1}, z_{2}$, we have

$$
\begin{gathered}
\operatorname{Cap}_{p}(\gamma, \partial \Omega ; \Omega) \sim d_{p}\left(z_{1}, z_{2}\right) \sim d_{\operatorname{Cap}_{p}}\left(z_{1}, z_{2}\right) \\
\sim \int_{\gamma} \operatorname{dist}(z, \partial \Omega)^{1-p} d z
\end{gathered}
$$

where the constants depend only on $p$.

We note that, in [22] the relations between $n$-capacity metric and quasihyperbolic metric was studied via quasiconformal mappings in $\mathbb{R}^{n}$. With the two theorems above, we are able to read both sides of (1.2) in terms of capacities and reveal their relations. We prove it in the last section.

Corollary 1.4 Let $\Omega$ be a Jordan $W^{1, q}$-extension domain in the plane with $2<q<\infty$. Then for any two points $z_{1}, z_{2} \in \Omega$, there exists a curve $\gamma \subset \Omega$ joining $z_{1}$ and $z_{2}$ such that

$\int_{\gamma} \operatorname{dist}(z, \partial \Omega)^{\frac{1}{q-1}} \mathrm{~d} s(z) \lesssim\left|z_{1}-z_{2}\right|^{\frac{q-2}{q-1}}$,

where the constant depend only on the norm of the extension operator and $q$.

All the results above can be extended to the case where $\Omega$ is not Jordan but simply connected in the plane, via exhausting $\Omega$ by a sequence of Jordan domains. However, for the simplicity of the statement we omit it.

\section{Prerequisites}

We usually write the constants as positive real numbers $C(\cdot)$ with parenthesis including all the parameters on which the constant depends. The constant $C(\cdot)$ may vary between appearances, even within a chain of inequalities. By $a \sim b$ we mean that $b / C \leq a \leq C b$ for some constant $C \geq 2$.

For Euclidean spaces $\mathbb{R}^{n}$, we denote the distance of sets $A$ and $B$ by dist $(A, B)$, and the diameter of a set $A$ by $\operatorname{diam}(A)$. Given an interval $I$ in $\mathbb{R}$, we call a continuous map $I \rightarrow X$ a 
path and its image a curve; the image of an injective map we call an arc. We denote by $\ell(\gamma)$ the length of the curve $\gamma$. Furthermore, if $\gamma$ is an arc, then we refer to $\gamma[x, y]$ the subarc of $\gamma$ between points $x$ and $y$ in $\gamma$. The unit disk in $\mathbb{R}^{2}$ we denote by $\mathbb{D}$.

Recall that the image $\Gamma$ of an embedding $\S^{1} \rightarrow \mathbb{C}$ is called a Jordan curve and, by the Jordan curve theorem, the set $\mathbb{C} \backslash \Gamma$ has exactly two components, both homeomorphic to the (open) unit disk $\mathbb{D}$. The bounded components of $\mathbb{C} \backslash \Gamma$ are called Jordan domains. By the Riemann mapping theorem, for each Jordan domain $\Omega$ in $\mathbb{C}$, there exists a conformal $\operatorname{map} \mathbb{D} \rightarrow \Omega$. Moreover, given a Jordan domain $\Omega$ and a conformal map $\varphi: \mathbb{D} \rightarrow \Omega, \varphi$ has a homeomorphic extension $\overline{\mathbb{D}} \rightarrow \bar{\Omega}$ by the Caratheodory-Osgood theorem, see e.g. [17].

Recall that for points $z_{1}$ and $z_{2}$ in $\mathbb{D}$, their hyperbolic distance is

$d_{h}\left(z_{1}, z_{2}\right)=\inf _{\alpha} \int_{\alpha} \frac{2}{1-|z|^{2}}|\mathrm{~d} z|$,

where the infimum is over all rectifiable curves $\alpha$ joining $z_{1}$ to $z_{2}$ in $\mathbb{D}$. The hyperbolic geodesics in $\mathbb{D}$ are arcs of (generalized) circles that intersect the unit circle orthogonally.

Let $\Omega$ be a Jordan domain $\mathbb{C}$ with a base point $z_{0}$. Given $z \in \partial \Omega$ and $r>0$, we define the conformal annulus $A(z, r ; \Omega)$ by

$A(z, r ; \Omega)=\varphi\left(\left\{x \in \overline{\mathbb{D}}: r / 2<\left|x-\varphi^{-1}(z)\right|<r\right\}\right)$,

where $\varphi$ is the homeomorphic extension of a conformal map $\mathbb{D} \rightarrow \Omega$ satisfying $0 \mapsto z_{0}$. We supress again the role of the base point $z_{0}$ in the notation. Also for notational convenience we write $A\left(y_{i}, k\right)$ instead of $A\left(y_{i}, k ; \Omega\right)$ if the domain $\Omega$ in question is clear from the context.

For the conformal annuli, we have the following comparison lemma; the proof of the analog of it in [2, p. 645] gives our version with notational changes.

Lemma 2.1 Let $\Omega$ be a Jordan domain, $y_{1}, y_{2} \in \partial \Omega$, and let $\gamma$ be the hyperbolic geodesic in $\Omega$ joining $y_{1}$ and $y_{2}$. For each $k \in \mathbb{Z}_{+}$, let $\gamma_{i, k}=A\left(y_{i}, 2^{-k} ; \Omega\right) \cap \gamma$. Then

$\operatorname{diam}\left(\gamma_{i, k}\right) \sim \operatorname{dist}\left(\gamma_{i, k}, \partial \Omega\right) \sim \ell\left(\gamma_{i, k}\right) \sim \ell\left(\gamma_{i, k+1}\right)$.

Furthermore, if $\alpha$ is a curve in $A\left(z_{i}, 2^{-k}\right)$ joining components of $\partial A\left(z_{i}, 2^{-k}\right) \cap \Omega$, then

$\ell(\alpha) \gtrsim \ell\left(\gamma_{i, k}\right)$.

The constants of comparability in (2.1) and (2.2) are independent of $\Omega$, points $y_{1}$ and $y_{2}$, the parameter $k$, and the (tacitly omitted) base point $z_{0}$.

We can apply Lemma 2.1 to show the following capacity estimate
Lemma 2.2 With the assumptions and notation in Lemma 2.1, for every conformal annulus $A\left(y_{i}, k ; \Omega\right)$ with $\gamma_{i, k} \subset \gamma\left[z_{1}, z_{2}\right]$, one has

$\ell\left(\gamma_{i, k}\right)^{2-p} \lesssim \int_{A\left(y_{i}, k\right)}|\nabla u|^{p} d x$

for each $u \in \Delta\left(\partial \Omega \cap A\left(y_{i}, k ; \Omega\right), \gamma_{i, k} ; A\left(y_{i}, k ; \Omega\right)\right)$, where the constant depends only on $p$.

Proof Fix the domain $\Omega$ and let $q=\frac{p}{p-1}$. By Theorem 1.2, we only need to bound the $q$-capacity of (a part of) the outer boundary and (a part of) the inner boundary of the conformal annulus $A\left(y_{i}, k\right)$ inside one of the components $A$ of $A\left(y_{i}, k\right) \backslash \gamma_{i, k}$ from above by a multiple of $\ell\left(\gamma_{i, k}\right)^{2-q}$.

Let us fix one of the component $A$. Inside the topological rectangle $A$, via an argument similar to [14, Sect. 3.2], we can find two hyperbolic geodesics $\gamma_{1} \subset A\left(y_{i}, k\right)$ and $\gamma_{2} \subset A\left(y_{i}, k\right)$ joining $\partial \Omega$ to $\gamma_{i, k}$ such that

$\ell\left(\gamma_{1}\right) \sim \ell\left(\gamma_{2}\right) \sim \ell\left(\gamma_{i, k}\right)$,

and

$\operatorname{dist}_{\Omega}\left(\gamma_{1}, \gamma_{2}\right) \geq c_{1} \ell\left(\gamma_{i, k}\right)$,

where the constants are absolute. These two curves divide $A$ into three topological rectangles $A_{1}, A_{2}, A_{3}$, where $\partial A_{1} \cap \partial A_{2}=\gamma_{1}$ and $\partial A_{3} \cap \partial A_{2}=\gamma_{2}$. Then the following test function

$w(x)=\max \left\{0,1-\frac{1}{2 c_{1} \ell\left(\gamma_{i, k}\right)} \operatorname{dist}_{\Omega}\left(x, A_{1}\right)\right\}$

implies the desired estimate; observe that

$|\nabla w| \lesssim \ell\left(\gamma_{i, k}\right)^{-1}$.

Then the lemma follows from Theorem 1.2 since $|A| \lesssim \ell\left(\gamma_{i, k}\right)^{2}$.

A fundamental estimate is the $p$-capacity of a spherical annulus in the plane; see e.g. [10, pp. 35-37] for the proof.

Lemma 2.3 Let $0<r<R<\infty$. Then, for any $x \in \mathbb{R}^{2}$ and $p \in(1, \infty) \backslash\{2\}$, we have

$\mathrm{Cap}_{p}\left(B(x, r), S^{1}(x, R) ; B(x, R)\right)=C(p)\left(R^{\frac{p-2}{p-1}}-r^{\frac{p-2}{p-1}}\right)^{1-p}$. 


\section{Proof of Theorem 1.3}

Proof of Theorem 1.3 Let $\alpha \subset \Omega$ be an arbitrary curve joining $z_{1}$ and $z_{2}$. We extend the hyperbolic geodesic $\gamma$ to the boundary, and denote the two end points on $\partial \Omega$ by $y_{1}$ and $y_{2}$. The notation in Lemma 2.1 will be applied.

We first show that

$d_{\text {Cap }_{p}}\left(z_{1}, z_{2}\right) \sim d_{p}\left(z_{1}, z_{2}\right)$.

The discussion is divided into two cases.

Case $1 \quad z_{1}$ and $z_{2}$ are in the same or neighboring conformal annulus $A\left(y_{i}, 2^{-k}\right)$ for some $i \in\{1,2\}$. Then by Lemma 2.1, we have

$\int_{\gamma} \operatorname{dist}(z, \partial \Omega)^{1-p} d z \sim \ell\left(\gamma\left[z_{1}, z_{2}\right]\right)^{2-p} \sim \operatorname{Cap}_{p}(\gamma, \partial \Omega ; \Omega)$.

For an arbitrary curve $\alpha$, there are two sub-cases:

Case 1.1 If the curve $\alpha$ joining $z_{1}, z_{2}$ satisfies

$\frac{1}{8} \ell\left(\gamma\left[z_{1}, z_{2}\right]\right) \leq \operatorname{dist}(\alpha, \partial \Omega) \lesssim \ell\left(\gamma\left[z_{1}, z_{2}\right]\right)$,

meaning that $\alpha$ is contained in a Whitney type set with absolute constant by Lemma 2.1, and then the standard capacity estimate Lemma 2.3 gives

$\int_{\alpha} \operatorname{dist}(z, \partial \Omega)^{1-p} d z \sim \ell\left(\gamma\left[z_{1}, z_{2}\right]\right)^{2-p} \gtrsim \operatorname{Cap}_{p}(\alpha, \partial \Omega ; \Omega)$,

with the constant depending only on $p$. The other direction follows from a proof similar to the one of [21, Theorem 11.7].

Case 1.2 Suppose the curve $\alpha$ joining $z_{1}, z_{2}$ satisfies

$\frac{1}{8} \ell\left(\gamma\left[z_{1}, z_{2}\right]\right) \geq \operatorname{dist}(\alpha, \partial \Omega)$

By our assumption and Lemma 2.1 there is a subcurve $\alpha^{\prime} \subset \alpha$ such that

$\ell\left(\gamma\left[z_{1}, z_{2}\right]\right) \geq \operatorname{dist}\left(\alpha^{\prime}, \partial \Omega\right) \geq \frac{1}{16} \ell\left(\gamma\left[z_{1}, z_{2}\right]\right)$

and $\operatorname{diam}\left(\alpha^{\prime}\right) \sim \ell\left(\gamma\left[z_{1}, z_{2}\right]\right)$, where the constant is absolute. Hence the same reasoning as in the previous paragraph gives

$\int_{\alpha^{\prime}} \operatorname{dist}(z, \partial \Omega)^{1-p} d z \sim \ell\left(\gamma\left[z_{1}, z_{2}\right]\right)^{2-p} \sim \operatorname{Cap}_{p}\left(\alpha^{\prime}, \partial \Omega ; \Omega\right)$

with the constant depending on $p$. Since $\alpha^{\prime} \subset \alpha$, $\int_{\alpha^{\prime}} \operatorname{dist}(z, \partial \Omega)^{1-p} \mathrm{~d} z \leq \int_{\alpha} \operatorname{dist}(z, \partial \Omega)^{1-p} \mathrm{~d} z$

and

$\operatorname{Cap}_{p}\left(\alpha^{\prime}, \partial \Omega ; \Omega\right) \leq \operatorname{Cap}_{p}(\alpha, \partial \Omega ; \Omega)$.

Since we take infimum among the curves $\gamma$ in the both definitions of $p$-metric and $p$-capacity metric, respectively, we conclude the theorem in the special case.

Case $2 z_{1}$ and $z_{2}$ are not in the same or neighboring conformal annulus $\varphi\left(A\left(y_{i}, 2^{-k}\right)\right)$ for some $i \in\{1,2\}$. We may assume that $z_{1}$ and $z_{2}$ are on the boundary of some conformal annuli by our consequence of Case 1 . Then by the subadditivity of $p$-capacity we conclude that

$d_{\text {Cap }_{p}}\left(z_{1}, z_{2}\right) \leq \operatorname{Cap}_{p}(\gamma, \partial \Omega ; \Omega) \leq \sum_{\gamma_{i, k} \subset \gamma\left[z_{1}, z_{2}\right]} \operatorname{Cap}_{p}\left(\gamma_{i, k}, \partial \Omega ; \Omega\right)$

By Lemma 2.1 (with the notation there) and Lemma 2.3, on each conformal annulus we have

$\operatorname{Cap}_{p}\left(\gamma_{i, k}, \partial \Omega ; \Omega\right) \lesssim \operatorname{Cap}_{p}\left(\gamma_{i, k}, \partial \Omega ; \mathbb{R}^{2}\right) \lesssim \ell\left(\gamma_{i, k}\right)^{2-p}$

with the constant depending only on $p$. By Lemma 2.1 again we have

$$
\begin{aligned}
& \sum_{\gamma_{i, k} \subset \gamma\left[z_{1}, z_{2}\right]} \ell\left(\gamma_{i, k}\right)^{2-p} \lesssim \\
& \sum_{\alpha_{i, k} \subset \alpha} \int_{\alpha_{i, k}} \operatorname{dist}(z, \partial \Omega)^{1-p} \mathrm{~d} z \\
& \leq \int_{\alpha} \operatorname{dist}(z, \partial \Omega)^{1-p} \mathrm{~d} z .
\end{aligned}
$$

where $\alpha_{i, k}$ is the part of $\alpha$ in the corresponding annulus for some arbitrary $\alpha$ joining $z_{1}$ and $z_{2}$. All in all, we have

$d_{\text {Cap }_{p}}\left(z_{1}, z_{2}\right) \lesssim d_{p}\left(z_{1}, z_{2}\right)$

by the arbitrariness of $\alpha$, where the constant depends only on $p$.

For the other direction, let $u \in \Delta(\gamma, \partial \Omega ; \Omega)$ be absolutely continuous along almost every line segment. Then by Lemmas 2.1 and 2.2, we have

$$
\begin{aligned}
d_{p}\left(z_{1}, z_{2}\right) & \leq \int_{\gamma} \operatorname{dist}(z, \partial \Omega)^{1-p} \mathrm{~d} z \\
& \lesssim \sum_{\gamma_{i, k} \subset \gamma\left[z_{1}, z_{2}\right]} \ell\left(\gamma_{i, k}\right)^{2-p} \\
& \lesssim \sum_{i, k} \int_{A\left(y_{i}, k\right)}|\nabla u|^{p} \mathrm{~d} x \leq \int_{\Omega}|\nabla u|^{p} \mathrm{~d} x .
\end{aligned}
$$


Then by taking the infimum over $u \in \Delta(\gamma, \partial \Omega ; \Omega)$ we obtain the other direction. Thus (3.1) follows with the constant depending only on $p$.

The rest part of the theorem follows analogously according to the calculation above: For example, by choosing $\alpha$ as $\gamma$ in (3.2) one concludes via the calculation above that

$\operatorname{Cap}_{p}(\gamma, \partial \Omega ; \Omega) \lesssim \int_{\gamma} \operatorname{dist}(z, \partial \Omega)^{1-p} \mathrm{~d} z \lesssim \int_{\Omega}|\nabla u|^{p} \mathrm{~d} x$,

and then obtains

$\operatorname{Cap}_{p}(\gamma, \partial \Omega ; \Omega) \lesssim d_{\operatorname{Cap}_{p}}\left(z_{1}, z_{2}\right)$

by the arbitrariness of $u \in \Delta(\gamma, \partial \Omega ; \Omega)$. The other inequalities in Theorem 1.3 follow similarly.

\section{The proof of Corollary 1.4}

Proof of Corollary 1.4 Let us first consider the case $z_{1}, z_{2} \in \partial \Omega$. We claim that for these two points, there exists a curve $\gamma \subset \Omega$ such that

$\int_{\gamma} \operatorname{dist}(z, \partial \Omega)^{\frac{1}{1-q}} \mathrm{~d} s(z) \lesssim\left|z_{1}-z_{2}\right|^{\frac{q-2}{q-1}}$.

Let $\gamma$ be the hyperbolic geodesic joining $z_{1}$ and $z_{2}$. Notice that $z_{1}, z_{2}$ divide $\partial \Omega$ into two subarcs; namely $\Omega=\Gamma_{1} \cup \Gamma_{2}$. Then each $\Gamma_{i}$ with the hyperbolic geodesic $\gamma$ gives us a Jordan domain. Moreover, by applying an approximation argument, we generalize Theorem 1.2 in the case where $\gamma_{1}=\Gamma_{1}, \gamma_{3}=\Gamma_{2}$, and $\gamma_{2}$ and $\gamma_{4}$ there are just points $z_{1}$ and $z_{2}$, respectively.

Since $1-p=1 /(1-q)$, we have, by Theorem 1.3, (generalized) Theorem 1.2 and the subadditivity of $p$-capacity,

$$
\begin{aligned}
\int_{\gamma} \operatorname{dist}(z, \partial \Omega)^{\frac{1}{1-q}} \mathrm{~d} s(z) & =\int_{\gamma} \operatorname{dist}(z, \partial \Omega)^{1-p} \mathrm{~d} s(z) \lesssim d_{\operatorname{Cap}_{p}}\left(z_{1}, z_{2}\right) \\
& \sim \operatorname{Cap}_{p}(\gamma, \partial \Omega, \Omega) \\
& \lesssim \operatorname{Cap}_{p}\left(\gamma, \Gamma_{1} ; \Omega\right)+\operatorname{Cap}_{p}\left(\gamma, \Gamma_{2} ; \Omega\right) \\
& \lesssim\left(\operatorname{Cap}_{q}\left(z_{1}, z_{2} ; \Omega\right)\right)^{-\frac{1}{q-1}}
\end{aligned}
$$

For $q>2$, Morrey's inequality [4, 4.5.3] yields

$\operatorname{Cap}_{q}\left(z_{1}, z_{2} ; \mathbb{R}^{2}\right) \sim\left|z_{1}-z_{2}\right|^{2-q}$.

Since $\Omega$ is a $W^{1, q}$-extension domain, then

$\operatorname{Cap}_{q}\left(z_{1}, z_{2} ; \Omega\right) \sim \operatorname{Cap}_{q}\left(z_{1}, z_{2} ; \mathbb{R}^{2}\right) \sim\left|z_{1}-z_{2}\right|^{2-q}$,

with the constant depending only on the extension operator. Hence, by (4.1), $\int_{\gamma} \operatorname{dist}(z, \partial \Omega)^{\frac{1}{q-1}} \mathrm{~d} s(z) \lesssim\left|z_{1}-z_{2}\right|^{\frac{q-2}{q-1}}$.

Thus the claim follows.

Now for a pair of points $x_{1}, x_{2} \in \Omega$, if

$2\left|x_{1}-x_{2}\right| \leq \max \left\{\operatorname{dist}\left(x_{1}, \partial \Omega\right), \operatorname{dist}\left(x_{2}, \partial \Omega\right)\right\}$,

then the line segment joining $x_{1}, x_{2}$ satisfies the desired curve condition. On the other hand, if

$2\left|x_{1}-x_{2}\right| \geq \max \left\{\operatorname{dist}\left(x_{1}, \partial \Omega\right), \operatorname{dist}\left(x_{2}, \partial \Omega\right)\right\}$,

let $z_{1}, z_{2} \in \partial \Omega$ such that

$\operatorname{dist}\left(x_{1}, z_{1}\right)=\operatorname{dist}\left(x_{1}, \partial \Omega\right), \quad \operatorname{dist}\left(x_{2}, z_{2}\right)=\operatorname{dist}\left(x_{2}, \partial \Omega\right)$.

Then by letting $\alpha=\left[x_{1}, z_{1}\right] \cup \gamma \cup\left[x_{2}, z_{2}\right]$, where $\left[x_{1}, z_{1}\right],\left[x_{2}, z_{2}\right]$ denote the line segment joining $x_{1}, z_{1}$ and $x_{2}, z_{2}$, respectively, and $\gamma$ is the hyperbolic geodesic joining $z_{1}, z_{2}$. Then by our claim above with the assumption (4.2), we conclude that

$$
\begin{aligned}
\int_{\alpha} \operatorname{dist}(z, \partial \Omega)^{\frac{1}{q-1}} \mathrm{~d} s(z) & \leq\left(\int_{\left[x_{1}, z_{1}\right]}+\int_{\gamma}+\int_{\left[x_{2}, z_{2}\right]}\right) \operatorname{dist}(z, \partial \Omega)^{\frac{1}{q-1}} \mathrm{~d} s(z) \\
& \lesssim\left|x_{1}-z_{1}\right|^{\frac{q-2}{q-1}}+\left|z_{1}-z_{2}\right|^{\frac{q-2}{q-1}}+\left|x_{2}-z_{2}\right|^{\frac{q-2}{q-1}} \\
& \lesssim\left|x_{1}-x_{2}\right|^{\frac{q-2}{q-1}}
\end{aligned}
$$

where we used the fact that the triangle inequality and the assumption (4.2) yield

$\left|z_{1}-z_{2}\right| \leq\left|x_{1}-z_{1}\right|+\left|x_{1}-x_{2}\right|+\left|x_{2}-z_{2}\right| \leq 5\left|x_{1}-x_{2}\right|$.

Remark 4.1 When $p=q=2$, one may apply Theorem 1.2 to a Jordan $W^{1,2}$-extension domain to similarly show that, for any $z_{1}, z_{2} \in \Omega$, the hyperbolic curve $\Gamma$ joining them satisfies

$\int_{\Gamma} \operatorname{dist}(z, \Omega)^{-1} \operatorname{ds}(z)\left(\log \left(1+\frac{\left|z_{1}-z_{2}\right|}{\min \left\{\operatorname{dist}\left(z_{1}, \partial \Omega\right), \operatorname{dist}\left(z_{2}, \partial \Omega\right)\right\}}\right)\right)^{-1} \lesssim 1$,

provided $z_{1}, z_{2}$ are relatively far (compared with their distances to the boundary). This is the Gehring-Osgood characterization of quasidisks [6], and it is proven that a Jordan domain in the plane is a $W^{1,2}$-extension domain if and only if the domain is a quasidisk; see $[7-9,11]$. Hence one can also prove the necessity of a Jordan $W^{1,2}$-extension domain without using any test function. Moreover, this indicates the relations between Gehring-Osgood condition and (1.2) via Theorem 1.2, i.e. the left-hand side is comparable to certain capacity ( $p$-capacity for (1.2) and 2-capacity for GehringOsgood condition) inside the domain, and the right-hand side is comparable to the reciprocal of its dual capacity in the Euclidean space with the correct power. 
Acknowledgements The author would like to appreciate the anonymous referee for his careful reading and nice suggestions for organizing the paper.

Funding Open Access funding provided by ETH Zurich. The author was partially funded by the European Research Council under the Grant Agreement No. 721675 "Regularity and Stability in Partial Differential Equations (RSPDE)”.

\section{Compliance with ethical standards}

Conflict of interest The author states that there is no conflict of interest.

Open Access This article is licensed under a Creative Commons Attribution 4.0 International License, which permits use, sharing, adaptation, distribution and reproduction in any medium or format, as long as you give appropriate credit to the original author(s) and the source, provide a link to the Creative Commons licence, and indicate if changes were made. The images or other third party material in this article are included in the article's Creative Commons licence, unless indicated otherwise in a credit line to the material. If material is not included in the article's Creative Commons licence and your intended use is not permitted by statutory regulation or exceeds the permitted use, you will need to obtain permission directly from the copyright holder. To view a copy of this licence, visit http://creativecommons.org/licenses/by/4.0/.

\section{References}

1. Aikawa, H., Ohtsuka, M.: Extremal length of vector measures. Ann. Acad. Sci. Fenn. Math. 24(1), 61-88 (1999)

2. Bonk, M., Koskela, P., Rohde, S.: Conformal metrics on the unit ball in Euclidean space. Proc. Lond. Math. Soc. (3) 77(3), 635664 (1998)

3. Buckley, S., Koskela, P.: Criteria for imbeddings of Sobolev-Poincaré type. Int. Math. Res. Not. 18, 881-902 (1996)

4. Evans, L.C., Gariepy, R.F.: Measure Theory and Fine Properties of Functions. Studies in Advanced Mathematics. CRC Press, Boca Raton, FL (1992)

5. Gehring, F.W., Martio, O.: Lipschitz classes and quasiconformal mappings. Ann. Acad. Sci. Fenn. Ser. A I Math. 10, 203-219 (1985)
6. Gehring, F.W., Osgood, B.G.: Uniform domains and the quasihyperbolic metric. J. Analyse Math. 36(1979), 50-74 (1980)

7. Gol'dshteı̌n, V.M., Latfullin, T.G., Vodop'yanov, S.K.: A criterion for the extension of functions of the class $L_{2}^{1}$ from unbounded plain domains. Sibirsk. Mat. Zh. 20, 416-419 (1979). (Russian)

8. Gol'dshteĭn, V.M., Reshetnyak, YuG: Quasiconformal Mappings and Sobolev Spaces, Mathematics and its Applications (Soviet Series). Kluwer Academic Publishers Group, Dordrecht (1990)

9. Gol'dshteřn, V.M., Vodop'yanov, S.K.: Prolongement de fonctions différentiables hors de domaines planes. C. R. Acad. Sci. Paris Ser. I Math. 293, 581-584 (1981). (French)

10. Heinonen, J., Kilpeläinen, T., Martio, O.: Nonlinear Potential Theory of Degenerate Elliptic Equations. Unabridged Republication of the: Original, p. 2006. Dover Publications Inc, Mineola, NY (1993)

11. Jones, P.W.: Quasiconformal mappings and extendability of Sobolev functions. Acta Math. 47, 71-88 (1981)

12. Koskela, P.: Extensions and imbeddings. J. Funct. Anal. 159, 1-15 (1998)

13. Koskela, P., Pankka, P., Zhang, Y. R.-Y.: Ahlfors reflection theorem for p-morphisms. arXiv: 1912.09200

14. Koskela, P., Zhang, Y.R.-Y.: A density problem for Sobolev spaces on planar domains. Arch. Ration. Mech. Anal. 222(1), 1-14 (2016)

15. Koskela, P., Rajala, T., Zhang, Y. R.-Y.: A geometric characterization of planar Sobolev extension domains.

16. Lappalainen, V.: $\mathrm{Lip}_{h}$-extension domains. Ann. Acad. Sci. Fenn. Ser. AI Math Diss. 56, 1-52 (1985)

17. Palka, B.: An Introduction to Complex Function Theory, Undergraduate Texts in Mathematics. Springer, New York (1991)

18. Rajala, K.: Uniformization of two-dimensional metric surfaces. Invent. Math. 207(3), 1301-1375 (2017)

19. Romanov, A.S.: Capacity relations in a planar quadrilateral. Sibirsk. Mat. Zh. 49(4), 886-897 (2008)

20. Shvartsman, P.: On Sobolev extension domains in $\mathbb{R}^{n}$. J. Funct. Anal. 258(7), 2205-2245 (2010)

21. Väisälä, J.: Lectures on n-Dimensional Quasiconformal Mappings. Lecture Notes in Mathematics, vol. 229. Springer, Berlin (1971)

22. Vuorinen, M.: Conformal invariants and quasiregular mappings. J. Analyse Math. 45, 69-115 (1985)

23. Ziemer, W.P.: Extremal length and conformal capacity. Trans. Am. Math. Soc. 126, 460-473 (1967)

Publisher's Note Springer Nature remains neutral with regard to jurisdictional claims in published maps and institutional affiliations. 